EPJ Web of Conferences 116, 01004 (2016)

DOI: $10.1051 /$ epjconf/201611601004

CC Owned by the authors, published by EDP Sciences, 2016

\title{
VSiPMT a new photon detector
}

\author{
F. Di Capua ${ }^{1,2, a}$, G. Barbarino ${ }^{1,2}$, F.C.T. Barbato ${ }^{1,2}$, L. Campajola ${ }^{1,2}$, R. de Asmundis ${ }^{2}$, G. De Rosa ${ }^{1,2}$, \\ P. Migliozzi ${ }^{2}$, C.M. Mollo ${ }^{2}$, and D. Vivolo ${ }^{1,2}$
}

${ }^{1}$ Physics Department, University of Naples, Napoli, Italy

2 INFN, Sezione di Napoli, Napoli, Italy

\begin{abstract}
Photon detection is a key factor to study many physical processes in several areas of fundamental physics research. Focusing the attention on photodetectors for particle astrophysics, the future experiments aimed at the study of very high-energy or extremely rare phenomena (e.g. dark matter, proton decay, neutrinos from astrophysical sources) will require additional improvements in linearity, gain, quantum efficiency and single photon counting capability. To meet the requirements of these class of experiments, we propose a new design for a modern hybrid photodetector: the VSiPMT (Vacuum Silicon PhotoMultiplier Tube). The idea is to replace the classical dynode chain of a PMT with a SiPM, which therefore acts as an electron detector and amplifier. The aim is to match the large sensitive area of a photocathode with the performances of the SiPM technology.
\end{abstract}

\section{Introduction}

Photodetectors are widely used in many areas of fundamental physics research. Focusing on astroparticle physics experiments, up to the present the photon detection capability of photomultiplier tubes (PMTs) seems to be unrivalled. Anyway, they have several disadvantages: fluctuations in the first dynode gain make single photon counting difficult; the linearity is strongly related to the gain; the transit time spreads over large fluctuations; the need of voltage dividers increases failure risks and the power consumption.

The future generation of experiments will require further improvement in linearity, gain, and sensitivity (quantum efficiency and single photon counting capability). So, even though PMTs represent a certified technology since 1936 [1], an alternative solution should be found.

The solid state photodetectors, APDs and SiPMs, represent a technological progress that goes beyond PMTs' limits. In particular, SiPM technology has achieved a very high level of performances but the size limited by the thermal noise partially compromises their application in astroparticle physiscs experiments.

The realization of a new photon detector with photoelectron multiplication based on SiPMs, having a photocathode of at least $1 \mathrm{inch}$, will have a tremendous impact on astroparticle physics experiment.

\footnotetext{
ae-mail: dicapua@na.infn.it
}

This is an Open Access article distributed under the terms of the Creative Commons Attribution License 4.0, which permits unrestricted use, distribution, and reproduction in any medium, provided the original work is properly cited. 


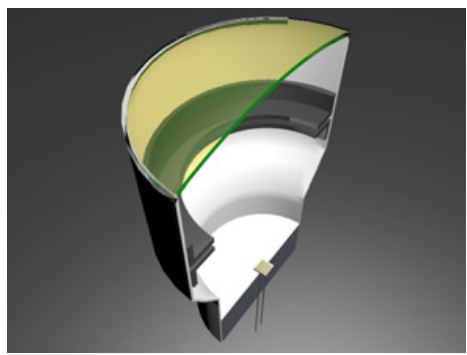

Figure 1. A cutaway of the VSiPMT showing the interior composition of the device.
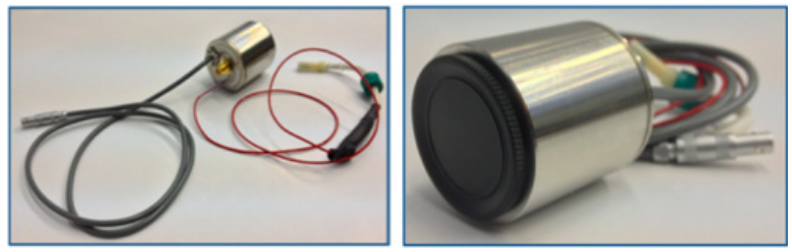

Figure 2. The VSiPMT prototype by Hamamatsu.

\section{A new high gain photodetector: The VSiPMT}

The central point of the challenge is finding a way to detect photons from large surfaces volumes as typically needed in many astroparticle experiments. An interesting solution is represented by VSiPMT (Vacuum Silicon PhotoMultiplier Tube), a new design for a modern hybrid photodetector based on the combination of a SiPM with an hemispherical PMT standard envelope.

The main idea, born in Naples in 2007 is to replace the classical dynode chain of a PMT with a SiPM, the latter acting as an electron multiplier at low voltage [2], see Fig. 1.

For this purpose a special no-windowed SiPM is necessary to allow the electrons to enter into the Silicon.

The VSiPMT feasibility study started with testing of a special non-windowed SiPM by Hamamatsu to an electron beam. The results[4] obtained encouraged Hamamatsu Photonics to realize two VSiPMT prototypes (Fig. 2).

The replacement of the divider makes the device compact and with three connections only: two cables to supply power to the photocathode and to the SiPM and one output for signal readout.

The prototypes have an envelope with a $7 \times 7 \mathrm{~mm}^{2}$ borosilicate glass entrance window and a GaAsP photocathode with a $3 \mathrm{~mm}$ diameter and a spectral response in the range between 300 and $750 \mathrm{~nm}$.

\section{The VSiPMT prototype characterization}

The prototypes underwent many tests in our laboratories [6], in order to achieve a full characterization of the devices.

A picosecond pulsed laser emitting in blue region $(407 \mathrm{~nm})$ is sent trough a system of optical fibres to the VSiPMT, its intensity of light is varied by using differently calibrated filters and is continuously monitored by a power meter. 


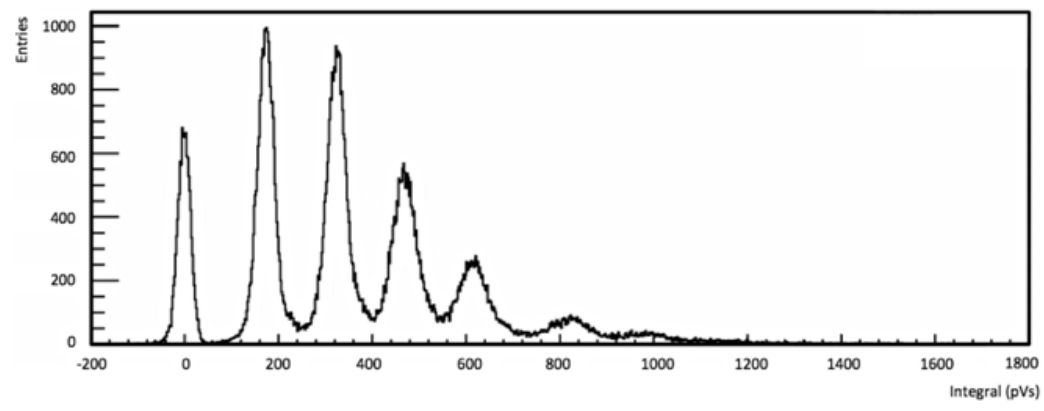

Figure 3. A typical VSiPMT charge spectrum.

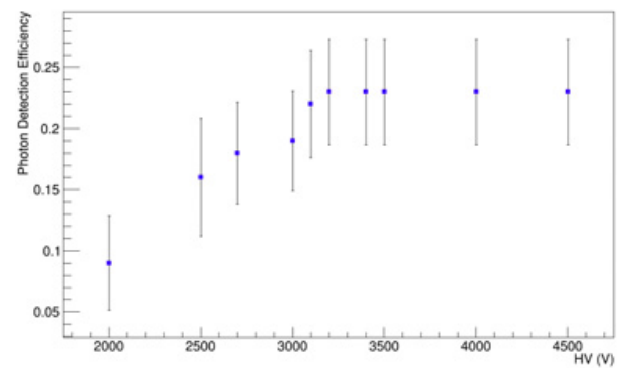

Figure 4. Results of the photon detection efficiency test in function of the HV. The PDE errors are obtained by the propagation of the errors on $N_{P E}$ and $N_{P H}$.

\subsection{Signal quality}

A typical charge spectrum of VSiPMT is shown in Fig. 3. The remarkable separation between the peaks demonstrate the excellent photon counting capability of such device. The peak-to-valley ratio has been measured to be about 60 in the single photon condition. The device exhibits an excellent gain stability (better than 2\%) in time.

\subsection{Detection efficiency and stability}

The device detection efficiency has been measured illuminating the device with few photons per pulse $(\sim 70)$ by using an optical fibre. Defining the Photon Detection Efficiency as

$$
P D E=\frac{N_{P E}}{N_{P H}}
$$

where $N_{P E}$ is the number of fired cells and $N_{P H}$ is the number of photons per laser pulse hitting the photocathode. As shown in Fig. 4 the PDE become stable around $-3 \mathrm{kV}$ with a plateau where a PDE value of about $23 \%$ has been found.

The PDE value measured is in agreement with expectation formula

$$
P D E_{V S i P M T}=Q E \times \epsilon_{S i P M} \times \epsilon_{\text {coll }}
$$

where QE is the photocathode quantum efficiency, $\epsilon_{S i} P M$ is the SiPM electron detection efficiency and $\epsilon_{\text {coll }}$ is the collection efficiency of the photoelectrons on the SiPM surface $\left(Q E \sim 0.38, \epsilon_{S i P M} \sim 0.61\right.$, $\left.\epsilon_{\text {coll }} \sim 1\right)$. 


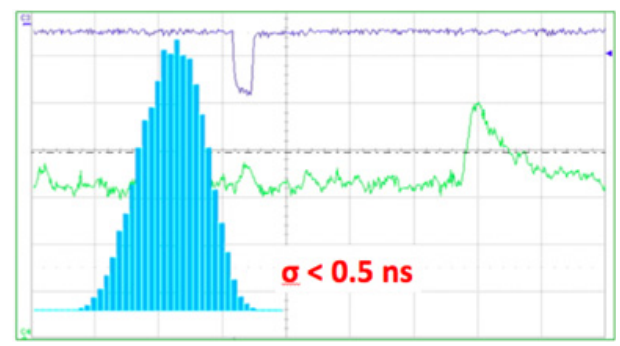

Figure 5. Printscreen of the oscilloscope. The blue squarewave is the trigger signal from the oscilloscope. The green waveform is the VSiPMT spe signal. The $\mathrm{x}$-axes unit is $20 \mathrm{~ns} / \mathrm{div}$ while the y-axes unit for the green line is $5 \mathrm{mV} / \mathrm{div}$. The histogram is the distribution of the arrival time of the VSiPMT signal with respect to the laser trigger.

In VSiPMT, the plateau region is linked to the energy threshold of the photoelectrons to enter into the the silicon bulk and, consequently, to produce a signal. The high voltage in such device is required only to drive photoelectrons to the SiPM surface and to give them the right energy to trigger the geiger avalanche.

\subsection{Gain}

As for a SiPM the gain in this device is the ratio between the single pixel charge output signal and the electron charge. As expected we found that the gain is totally independent of the high voltage and we measured for SiPM bias voltage set to $72.5 \mathrm{~V}$ a value of $5.6 \times 10^{5}$.

\subsection{Transit Time Spread}

We expect the TTS to be smaller for the VSiPMT than for a standard PMT. For a standard PMT, indeed, there are two contributions to the TTS: one is due to the different trajectories of the photoelectrons going from the photocathode to the first dynode, the other is due to the different trajectories of the seconday electrons between the dynode chain. In the VSiPMT the dynode chain is replaced by a SiPM, thus for such a device the TTS is simply given by first contribution. Taking into account an eventual laser jitter, the TTS upper limit has been estimated to be $<0.5$ ns (Fig. 5).

\section{Summary of VSiPMT performances and conclusions}

The VSiPMT project born in the University of Naples in 2007 to provide good alternative to PMTs. The simple substitution of the dynode chain with a SiPM allows to reach a very high gain totally provided by the pixels working in geiger mode.

The devices show outstanding properties and performances beyond expectations, such as: excellent photon counting capability, fast response, low power consumption and great stability. VSiPMT linearity follows the same behaviour as for SiPM with the possibility to play with the number of cells in order to vary the dynamic range.

All these attractive features lead us to go ahead with a second phase aimed at the realization of a new optimized and usable version of the prototypes.

We are now attempting to realize a prototype with a larger photocatode and study an optimized configuration for a better electron focusing in order to fire full SiPM and, consequently, improve the dynamic range of the device.

Moreover in the future a SiPM optimized for electron multiplier could be designed. In fact in contrast with the photon detection, the precise and very short range of the photoelectrons incoming in SiPM, 
in which few electron-hole pairs are enough to induce the micro-pixel Geiger discharge, permits the reduction of the thickness of the junction $\mathrm{p}+\mathrm{nn}+$ layers with great advantage for lowering the dark current, increasing at same time the efficiency and the time resolution. Definitive and commercial VSiPMT will be constructed in synergetic collaboration with specialized factories and semiconductor research laboratories.

\section{References}

[1] H. Iams, B. Salzberg, The secondary emission phototube, Proceedings of the Institute of Radio Engineers, 1935, 0731-5996

[2] G. Barbarino et al., A new high-gain vacuum photomultiplier based upon the amplification of a Geiger-mode p-n junction, Nucl. Instrum. Meth. A, 2008, 594, 326-331

[3] G. Barbarino et al., VSiPMT for underwater neutrino telescopes, Nucl. Instrum. Meth. A, 2013, 725, $162-165$

[4] G. Barbarino et al., Proof of feasibility of the Vacuum Silicon PhotoMultiplier Tube (VSiPMT), JINST, 2013, 8, P04021

[5] C. Joram et al., Proof of principle of G-APD based hybrid photodetectors, Nucl. Instrum. Meth. A, 2010, 621(1-3) 171-176

[6] G. Barbarino et al., A new generation photodetector for astroparticle physics: the VSiPMT, Astroparticle Physics Journal, 2015, 67, 18-25 